\title{
Design of Space for Expression Media with the Use of Fog
}

\author{
Shiroh Itai ${ }^{1}$, Yuji Endo ${ }^{2}$, and Yoshiyuki Miwa ${ }^{1}$ \\ ${ }^{1}$ Faculty of Science and Engineering, Waseda University, Tokyo, Japan \\ itai@aoni.waseda.jp, miwa@waseda.jp \\ ${ }^{2}$ Graduate School of Creative Science and Engineering, Waseda University, Tokyo, Japan \\ y-endo@akane.waseda.jp
}

\begin{abstract}
In this research, we designed and developed a "playground" using fog as a media of expression. The "playground" is a space where children express themselves and connect with others using bodily expressions while playing. To realize this concept, we developed the fog display system to satisfy the following requirements. (1) Everyone has access to the media space created by the fog. (2) Fog displays are scattered all over the space. (3) Images projected on fog displays are visible from various directions inside and outside the space. (4) The expelling, rectification, and diffusion of the fog are controlled. And, we tested the effectiveness of the system through its on-site use. As a result, it was found that this system, which can project an image on the fog that has a naturalness (disappearance, spatiality, and extraordinariness), versatility, and plasticity, has the potential to function as the playground.
\end{abstract}

Keywords: Bodily expression, Fog, Playground, Open media space.

\section{Introduction}

In modern society, there has been a remarkable decline in communication skills and the ability to express oneself among children and the young. Furthermore, the opportunity to meet and share experiences with others through expression is becoming increasingly rare. Reports claim that those with more experience with playing during their childhood, and those with more natural experience, tend to have higher levels of motivation, more purpose in life, and better moral and relationshiprelated skills [1]. Thus, we have investigated assistive technology for extracting bodily expressions through media in order to connect individuals with each other through co-creation of expression [2-5]. In this study, we aim to create a "playground" using media technology for children to create bodily expressions while they play so as to connect with others through their expressions.

To realize this, the authors have focused on the sandbox, which is considered to be one of the best examples of a playground [6]. Characteristics of a sandbox include an "open space" that is "freely accessible by anyone," and a place that allows "improvised play that is produced naturally through embodied interaction." In this context, Minowa have pointed out that sand has the versatility and plasticity to create a variety of playing styles [7]. Based on the above, we suggest that creating a 
playground using media technology requires incorporating the function of the audience and nature by opening normally closed media spaces outward, and making use of media with versatility and plasticity.

In this study, we have decided to carry out the design and development of a media space by utilizing the properties of fog. Because people can move through the fog and look through to the other side, we think the opened media space can be realized if images are projected onto the fog. In addition, because of its plasticity and versatility, it is possible for fog to produce complex movements that cannot be predicted by humans through changes in the environment, such as the flow of the air and human touch. For this reason, interaction through fog can allow children to play in a way that is akin to playing in a sandbox, which would allow them to draw out the full originality and creativity of such play. Thus, in this study, we have carried out design and development of a playground by media expression through fog.

A potential issue in carrying out this study is that the media technology to be developed in this study has an inseparable relationship with the children's playgrounds where the technology will be utilized. Therefore, in this study, the engineers have attempted to proceed with device development by bringing the proposed device into the field where play occurs, and reflecting the insights obtained in the design of the device.

Specifically, the fog display [8] we developed was first modified so that it could be brought into the field of play and used by children. Then, based on these results, the playground we designed and developed was arranged in a three-dimensional fog display, and utilized in actual children's playgrounds. The details are reported below.

\section{Development of a Portable Fog Display and Its Hands-On Display}

The fog display [8] we developed has exhibited problems including: (1) The display is large and heavy, (2) the plane of projection is small, and (3) the device can get the user wet due to water leakage. Thus, it has proved difficult to bring the device to the children's field of play. Therefore, we have developed a new portable fog display consisting of a fog creation unit and fog-expelling unit, which has resolved these issues.

The fog creation unit utilizes an ultrasonic atomizer capable of generating fine fog in the tank and sends it to the fog-expelling unit using the blast of a sirocco fan. The fog-expelling unit is comprised of several fog-expelling devices (width $446 \times$ depth $306 \times$ height 235 [mm], weight $4.7 \mathrm{~kg}$ ). This unit is made of an acrylic material that is $3 \mathrm{~mm}$ thick, and was made compact and lightweight so that it could be carried by one person by setting the distance between the inlet and outlet ports at $235 \mathrm{~mm}$. In addition, placing a neodymium magnet (length $10 \times$ width 10 , thickness $2[\mathrm{~mm}]$ ) on the side of this unit has facilitated the combination and separation of the unit. By producing the above fog-expelling unit, we have solved problem (1) and eased the transportation and installation of the device to the site. Next, in order to solve problem (2), an air outlet $(446 \times 20 \mathrm{~mm})$ was set up on both sides of the fog expeller $(440 \times$ $140 \mathrm{~mm}$ ) to sandwich the fog in layers of air (air curtains). 


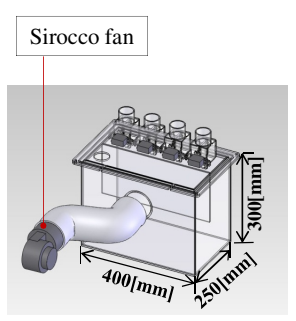

Fog creation unit

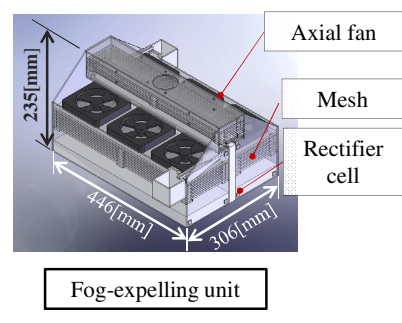

Fog-expelling unit

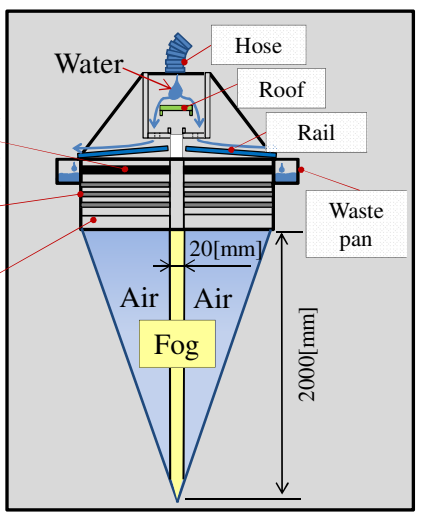

Lateral view of fog-expelling unit

Fig. 1. Fog-expelling device

A swirl flow of an axial flow fan installed to generate the air curtains and the flow of the air curtains is regulated by passing them through a mesh (core size: $4 \times 4[\mathrm{~mm}]$ ) and a rectifier cell. By sandwiching the fog in the air curtains, the fog is not diffused but injected into a layer $446 \mathrm{~mm}$ in width and $20 \mathrm{~mm}$ in thickness. As a result, the imaging size of one fog-expelling device was $446 \mathrm{~mm}$ in width and $2000 \mathrm{~mm}$ in height. Furthermore, a water leakage prevention mechanism was installed to solve problem (3). Specifically, as shown in Fig. 1, a function was added to drain the water that accumulated in the fog-expelling unit towards the water receptacle outside the unit to prevent the water from falling onto the user.

This hands-on display was shown at the Marunouchi Kids Festa (August 15-16, 2011, Tokyo International Forum) and it was intended that the children take advantage of this device in places where they actually play. As for the device composition, four fog-expelling units were connected longitudinally to generate a fog display $1800 \mathrm{~mm}$ in width and $2000 \mathrm{~mm}$ in height. We also created content that the children could play with by directly touching and interacting with the image projected on the fog display. More specifically, the skeleton data of the user is obtained with Kinect, and based on the position data of the skeleton (head, hands, and feet), the moving trajectory of the right hand touching the fog is left on the fog display as a stroke which can then be moved with the left hand (Fig. 2). In addition, visual representation software has been developed to extend the ripples from the position where the user has touched the fog. The aforementioned fog display system was experienced by more than 300 infants, elementary school students, and parents. As a result, a variety of activities were observed with the fog display, including: 1) situations where they were able to recall/imagine images such as "heart shapes," or "rocket shapes flying above," that they were then able to draw, or 2) collaborations between two or more participants working together to create one object (Fig. 3(a)). 


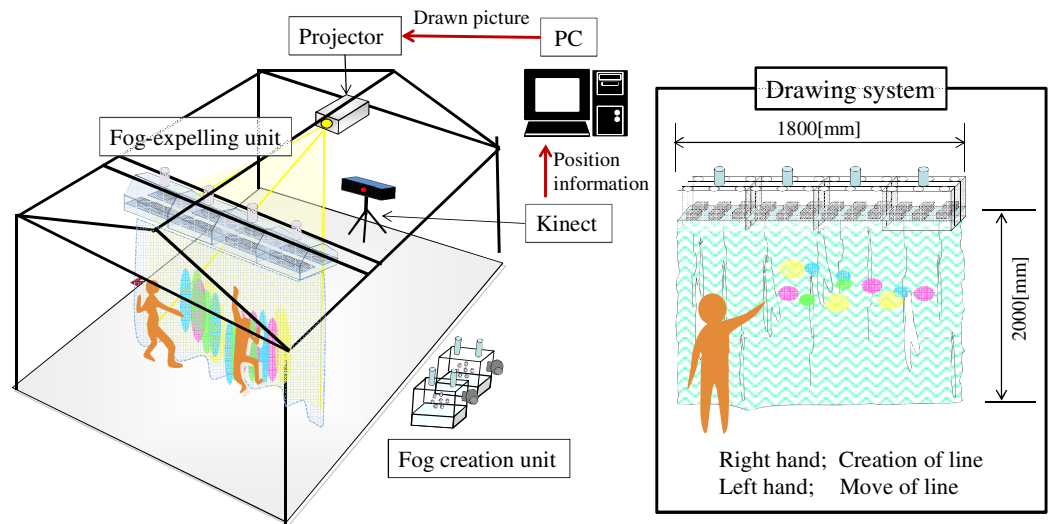

Fig. 2. Drawing system by Fog Display

This has confirmed the possibility that this system can be used to draw out body movements and thus support communication between users. In addition, it is interesting to note that users exhibited behaviors suggesting that they seemed to enjoy touching the fog as well as interacting with images (Fig. 3(b)). Specifically, they seemed to enjoy activities such as passing through the fog, grasping the fog with both hands, eating the fog, and bathing in the fog. This is an indication that the fog itself may allow for the creation of an image and draw out bodily expression. However, the direction of the body and movement is limited by the projection plane of the fog display, which in turn limits bodily expression. The system also gives the impression that the media space is closed off by the fog display. In other words, it was discovered that this system has not yet been able to fully achieve the open media space described in the previous section.
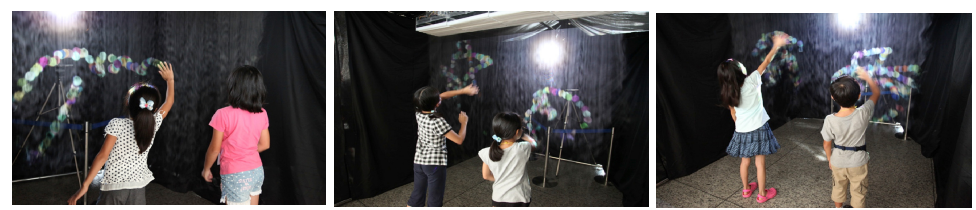

(a) Drawing in the fog display
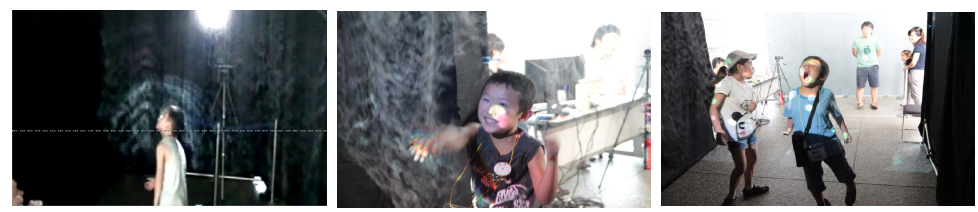

(b) Playing with fog itself

Fig. 3. Pictures of an interactive exhibition at the Kids Festa 


\section{Design and Development of a Playground through Media Expression with Fog}

Based on the results of the hands-on exhibition described in the previous section, we have conceived a playground through media technology using fog as shown in Fig. 4. In other words, in order to realize the open media, we attempt to create a space filled with fog which allows free access to anyone and construct an open media space by projecting images from various directions within this space. In addition, we attempt to change the shape and flow of the fog itself so that the users will be able to create images and bodily expressions through interaction with the fog. Thus, we aim at the realization of a playground where children, parents who watch their children and general visitors can meet and connect with each other by playing with the fog. This will be accomplished by allowing them to voluntarily move their bodies to create new bodily expressions and images.

In order to achieve this concept, we have set the following design requirements and developed a playground using fog:

(1) Anyone can come and go freely.

(2) Fog displays are scattered throughout the space.

(3) Images can be visually recognized from various directions both inside and outside the space.

(4) Expelling, rectification and diffusion of the fog can be freely controlled.

For (1) and (2), a space $2.5 \mathrm{~m}$ in width, $2.5 \mathrm{~m}$ in depth, and $2.0 \mathrm{~m}$ in height was set up assuming 3-5 children enter and exit freely. Sixteen fog-expelling units were then

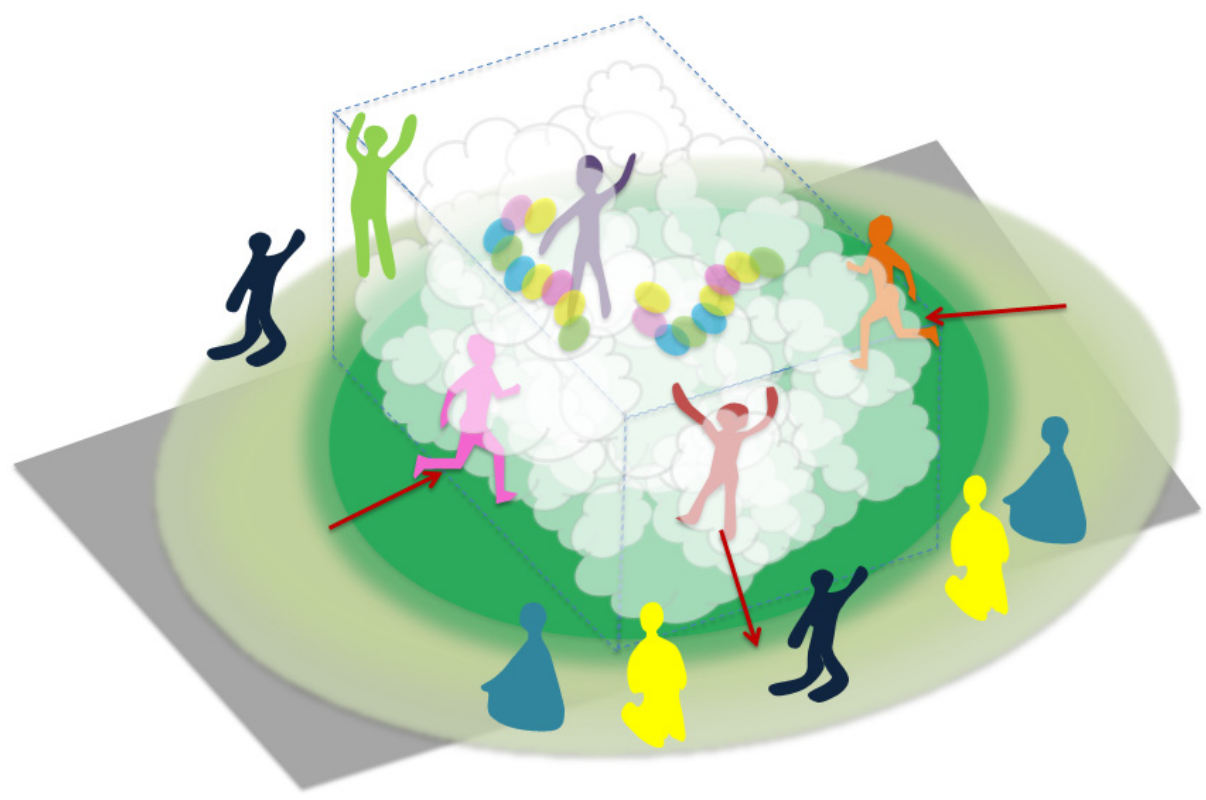

Fig. 4. Concept for "playground" 


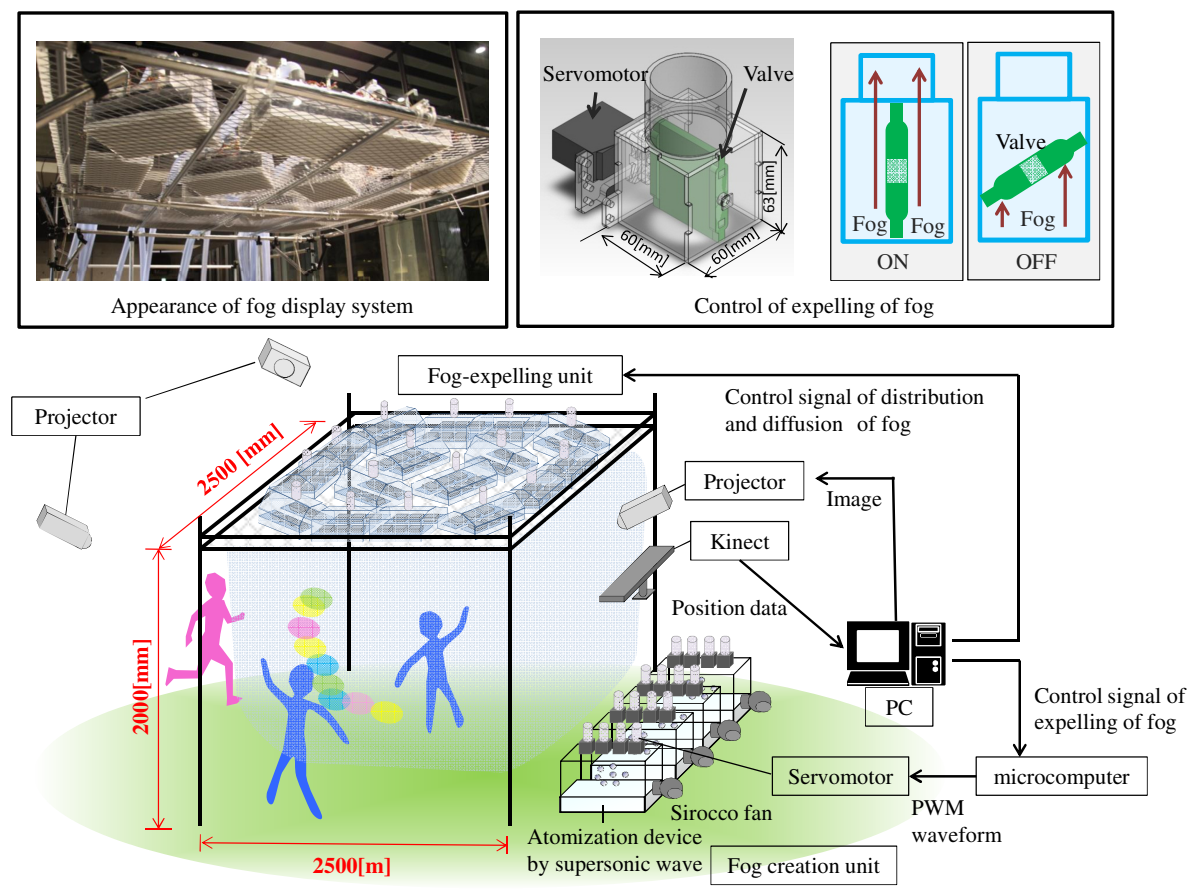

Fig. 5. Expression media space with the use of fog for Playground

placed concentrically in the upper part of this space (Fig. 5). An expanded metal was placed on top of the space (1200 mm horizontally x $1200 \mathrm{~mm}$ vertically) enabling the placement of fog-expelling units anywhere on it. Thus, by localizing in space the area where the fogs exists, we aim to diversify the space and support the creation of bodily expression that is not tied to the position and orientation of the fog display. Furthermore, four creation unit units were placed together on the back of one side of the fog space, allowing users to come and go freely in the fog space. Next, in order to achieve (3), images were projected into the fog space using three projectors as shown in Fig. 5. This allowed children and the audience watching them to visually recognize images projected onto the fog display from any direction both inside and outside the fog space. Furthermore, control of the amount of fog expelling was achieved by providing a valve at the supply outlets in the tank to each fog-expelling unit. Note that, in this system, the opening and closing of the valve is performed using a servo motor. Expelling of the rectified and diffused fog has been achieved by turning the air curtains in the fog-expelling unit on and off with a relay circuit.

In addition, the following four items represent content that could be played within the fog space:

(a) Projecting CG and scenery images into the fog space.

(b) Interacting with images in the fog space.

(c) Creating media expressions with the fog by controlling its expelling, rectification, and diffusion.

(d) Exploring the fog space using a light. 
By combining the above content, we aim to help the users make new discoveries and create new ways to play through both the creation of mental pictures from images and interaction with the fog.

\section{$4 \quad$ Hands-On Display of a Playground through Media Expression with Fog}

We conducted a hands-on exhibition at the National Research Center for Child Health and Development (January 30-February 2, 2012, Tokyo) where parents and the children admitted to this center were able to actually play with the system. This exhibition was experienced by more than 400 people ranging from children to adults. Fig. 6 shows pictures for this exhibition. As the fog is sterically-expelled in a space, we were able to observe the children moving around the entirety of the space (Fig. 6(a)). Since the fog space is open to the outside, parents, guardians and the children they were watching were able to talk to each other, enter and exit the space, and move

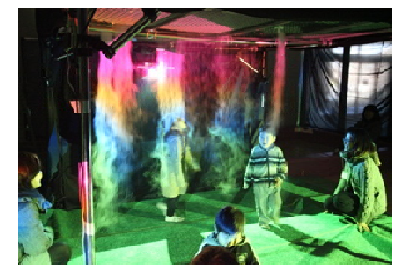

(a) Children bouncing off the walls throughout the whole space

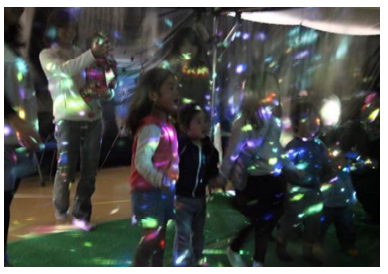

(c) Children in motion in tune with the CG's motion

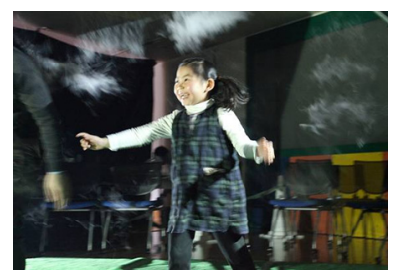

(e) Children chasing the intermittently expelled fog
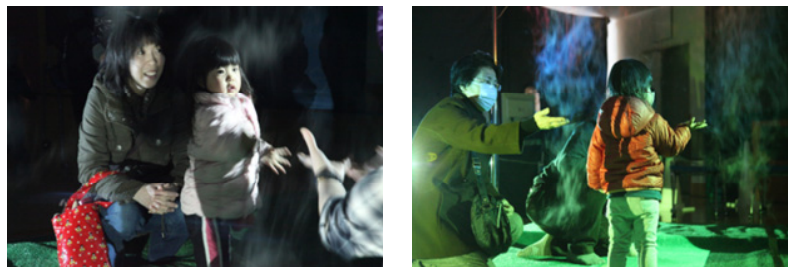

(b) Children and their parents playing together
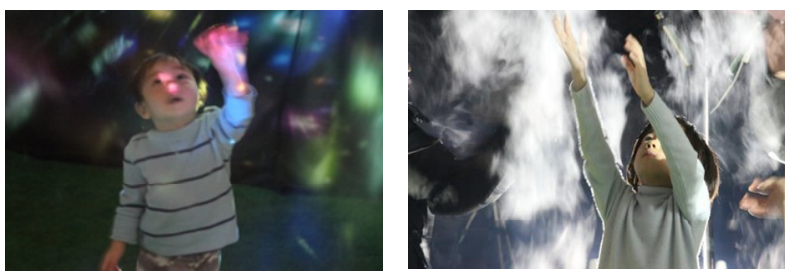

(d) Children touching the diffused fog
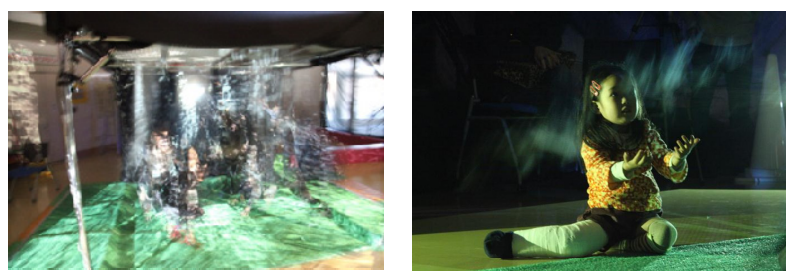

(f) Disabled children touching the fog

Fig. 6. Pictures of an interactive exhibition at the National Center for Child Health and Development 
their bodies freely (Fig. 6(b)). In addition, since it is possible to visually recognize the three-dimensionally generated image from any direction, we were able to observe the children making bodily expressions in various directions and jumping in response to the movement of the CG image (Fig. 6(c)). The children also created new methods of play by utilizing the fog itself, such as touching the diffused for everyone (Fig. 6(d)), moving around, or chasing the intermittently expelled fog (Fig. 6(e)). Children with cerebral palsy and leg injuries were observed reaching out towards the fog (Fig. 6(f)), voluntarily talking to other children in their surroundings, and moving cooperatively with them. Caregivers that were present made comments such as "The children playing in the fog were full of smiles, not showing signs of their diseases at all" and "It was easy for us to move around with the children according to the image projected in the fog." These results show that bodily expression and communication of children are produced and lively play is created from images projected in the fog space. It is also believed that, by taking advantage of the fog space like this, a connection between people can be created regardless of age, gender, and the presence or absence of disorders.

\section{$5 \quad$ Summary}

In this study we constructed an open media space using fog and carried out the design and development of a playground that supports user body expression and the creation of communication. First, we developed a fog display system that can be taken out into the field and carried out a hands-on exhibition of this system at the Marunouchi Kids Festa. Using the results from this hands-on exhibition, we then conducted the design and development of a space for expression media using the fog and incorporated it into the playground. Specifically, we placed 16 fog-expelling units concentrically that can control the amount of fog expelling, and developed a system that can project an image from multiple directions to the fog expelled from these units. We utilized this system at the National Research Center for Child Health and Development and observed children who created images and produced bodily expressions and communication. This allowed us to confirm the prospect that this system can function as a playground. In addition, these results show that play can be drawn out of children by creating a media expression and interacting with fog which has naturalness (disappearance, spatiality, and extraordinariness), versatility, and plasticity. In the future, we would like to further study design methods for a playground that takes advantage of media by focusing on the naturalness of the fog and considering media expression methods that can further extract these function of fog.

Acknowledgments. This study was conducted under the project "Generation and Control Technology of Human-Entrained Embodied Media" and is supported by CREST of JST, Project Research "Principal of emergence for empathetic "Ba" and its applicability to communication technology" by RISE Waseda University, and the GCOE program "Global Robot Academia." We would like to thank Dr. Hidekazu Masaki (National Center for Child Health and Development), Prof. Hiroko Nishi (Toyo Eiwa University), and Dr. Takabumi Watanabe (Waseda University) for their valuable suggestion during the course of this project and conducting the experiment. 


\section{References}

1. National Institution For Youth Education: Survey research about the reality of children's experience activity (2010) (in Japanese)

2. Miwa, Y., Itai, S., Watanabe, T., Iida, K., Nishi, H.: Shadow awareness: Bodily expression supporting system with use of artificial shadow. In: Jacko, J.A. (ed.) HCII 2009, Part II. LNCS, vol. 5611, pp. 226-235. Springer, Heidelberg (2009)

3. Iida, K., Itai, S., Nishi, H., Miwa, Y.: Utilization of shadow media - supporting co-creation of bodily expression activity in a group. In: Smith, M.J., Salvendy, G. (eds.) HCII 2011, Part I. LNCS, vol. 6771, pp. 408-417. Springer, Heidelberg (2011)

4. Miwa, Y., Itai, S., Watanabe, T., Nishi, H.: Shadow Awareness: Enhancing theater space through the mutual projection of images on a connective slit-screen. Leonardo, The Journal of the International Society for the Arts, Sciences and Technology 44(4), 325-333 (2011)

5. Osaki, A., Taniguchi, H., Miwa, Y.: Collaborative Aerial-Drawing System for Supporting Co-Creative Communication. Journal of Advanced Mechanical Design, Systems and Manufacturing 3(1), 93-104 (2009)

6. Fulghum, R.: All I Really Need to Know I Learned in Kindergarten. Ballantine Books (2004)

7. Minowa, J.: Allure of sandbox. Education of Infant Child 108(5), 13-17 (2009) (in Japanese)

8. Endo, Y., Inazawa, R., Maeda, K., Itai, S., Miwa, Y.: Development of 3D display device by multi-layered structuration of fog screens. In: Proceeding of Human Interface Symposium 2010, CD-ROM (2010) (in Japanese) 